\title{
HACIA UN CONCEPTO AMPLIO DE MAGNITUD ESCALAR
}

\author{
TOWARDS A BROAD CONCEPT OF SCALAR MAGNITUDE
}

\author{
Alejandro Sobrino \\ (Universidad de Santiago de Compostela, España) \\ alejandro.sobrino@usc.es \\ Enric Trillas Ruiz \\ (Universidad Politécnica de Madrid, España) \\ etrillasetrillas@gmail.com
}

Recibido: 21/11/2017

Aprobado: 19/12/2017

\begin{abstract}
RESUMEN
Se propone una ampliación del concepto de magnitud escalar para incluir aquello que puede ser poseído en un grado por los objetos y que no es aditivo. Tal ocurre con la propiedad 'grande' o con la medida de 'posibilidad', que es sub-aditiva. Se identifican los conceptos borrosos como magnitudes escalares, se muestra el carácter plural de su medida que no es única y se obtiene por criterios contextuales de diseño. Por último, se alude a las medidas de borrosidad de De Luca y Termini como magnitudes escalares que parten de la relación 'menos borroso que'.

Palabras clave: magnitud escalar, predicados vagos, medidas de borrosidad.

ABSTRACT

In this paper we will approach an extension of the concept of scalar magnitude in order to include the properties that can be attributed in a degree to the objects and that are not additive. That is the case of the property 'big' or the measure of 'possibility', which is subadditive. Fuzzy concepts are identified as scalar magnitudes and the plural character of their measure is emphasized, showing that is not unique and contextual design dependent. Finally, the fuzzy measures of De Luca and Termini are presented as scalar magnitudes based on the 'less fuzzy than' relation.
\end{abstract}

Key words: scalar magnitude, vague predicates, measures of fuzziness

\section{Introducción}

Muchos objetos o entidades de nuestro interés lo son porque, de alguna manera, podemos aprehenderlos. Una manera de hacerlo es determinando sus propiedades, cuantitativas o cualitativas, y midiéndolas. Conceptos físicos, como masa, velocidad, energía o volumen, se determinan usando entidades matemáticas, como números o vectores. Las propiedades cualitativas, en cambio, necesitan de nuevas herramientas matemáticas (Trillas, 2017). Las magnitudes físicas son susceptibles de ser medidas y sus medidas se realizan asignado números a las propiedades mensurables. Así, decimos que una persona pesa $70 \mathrm{~kg}$., que el volumen que aloja un frasco es de $50 \mathrm{ml}$., etc. En tanto indican valores posibles de esas magnitudes físicas, $50 \mathrm{~kg}$. Y $50 \mathrm{ml}$. son cantidades. 
Los sistemas cognitivos numéricos son una propiedad de los humanos que es compartida con otras especies animales; surgen en una etapa temprana del desarrollo, a los 4 años de edad, aunque experimentos con potenciales evocados sugieren que en los primeros meses de vida se hacen ya comparaciones. Múltiples estudios muestran que el sustrato cognitivo numérico se asienta, tanto en primates humanos como no humanos, en el córtex parietal de ambos hemisferios del cerebro y, más concretamente, en el surco interparietal (Cantlon; Platt; Brannon, 2007).

1.1. Usar entidades matemáticas para medir es un recurso adecuado, porque permiten capturar propiedades físicas del mundo y ciertas relaciones estructurales que se verifican entre ellas (Ellis, 1966). Como se ha dicho, a cada magnitud física le corresponde una clase de cantidades o valores. Pero además, se pueden realizar operaciones con ellas: p. ej., si se amplía el volumen de un frasco de $25 \mathrm{ml}$. en $10 \mathrm{ml}$. más, aloja hasta $35 \mathrm{ml}$. Se dice entonces que la medida de volúmenes es aditiva.

Las relaciones entre magnitudes físicas medibles o entre instancias de ellas pueden ser de dos tipos: métricas y sub-métricas. En las métricas, la relación se establece en términos numéricos (el canon de belleza griego indica que el cuerpo humano, para ser perfecto, debe medir 7 veces la medida de la cabeza) y en las sub-métricas, en términos simplemente comparativos. En cualquier caso, ambas relaciones permiten establecer un orden entre objetos. Si < denota a la relación binaria 'menor que' entre cantidades $\mathrm{m}$ de alguna magnitud $\mathrm{M}, \mathrm{m}(\mathrm{a})<\mathrm{m}$ (b) indica que la magnitud de $a$ es menor que la magnitud de $b$. Si M es volumen, m (a) $<$ m (b) denota que el volumen de $a$ es menor que el volumen de $b$. Los objetos que presentan cantidades se pueden medir y ordenar respecto de cada medida.

Además de establecer un orden entre magnitudes, los valores se pueden sumar o concatenar. Si sube a un ascensor una persona que pesa $50 \mathrm{~kg}$. y acto seguido se sube otra persona que pesa $70 \mathrm{~kg}$., el ascensor debe desplazar $120 \mathrm{~kg}$. una vez unido a su propio peso, que se añadiría a los pesos anteriores. Cuando una cantidad resulta de las sumas repetidas del mismo número, denotamos su ratio; así, si el valor de m (b) es 1000 cuando el valor de m(a) es 100 o el valor de m (b) es 100 cuando el valor de m(a) es 10, la ratio es 1:10.

Accedemos al conocimiento de las entidades físicas clásicas efectuando medidas sobre ellas. La medida de una cantidad $\mathrm{M}$ es una acción llevada a cabo sobre un objeto que muestra $\mathrm{M}$ usando un instrumento de medida y que tiene como resultado la determinación de posibles valores, mutuamente incompatibles (posiciones diferentes de los platos de una balanza, distintos niveles de un líquido en un recipiente, etc.), para esa cantidad. Una medida es exitosa si corresponde a un orden entre las entidades medidas; para el caso del peso, p. ej., si se determina que el plato a está en una posición más alta que el plato $b$ en la balanza. Medir es esencial para domesticar científicamente los conceptos adquiridos por observación de la realidad.

1.2. Aunque la descripción anterior de medida parece general, no lo es. Distintas magnitudes físicas requieren diferentes formas de caracterizar a sus medidas. Eso se muestra si, en vez de describir una medida, tratamos de explicarla. En ese caso, magnitudes como masa o longitud se muestran diferentes. Mientras la medida de la masa ha de atender a leyes físicas de la naturaleza -leyes del movimiento y la gravitación-, la determinación de la longitud es fruto de una convención. En el primer caso, la medida se fundamenta en las propiedades cuantitativas del objeto, las leyes de la física y los instrumentos de medida, factores que, conjuntamente, determinan la 'masa' y la relación 'tener menos masa que'. En cambio, las propiedades cuantitativas de la longitud requieren de dos factores, propiedad cuantificable e instrumento de medida, y se necesitan convenciones para operar con esos instrumentos en condiciones de objetividad. Usualmente se establece un patrón, y cualquier objeto es medido con referencia a él: la medida debe determinar la relación que hay entre el objeto y el patrón: si es menor, igual o mayor a él, o en qué proporción lo es. $6 \mathrm{~cm}$. es mayor que $3 \mathrm{~cm}$. y es seis veces la concatenación de $1 \mathrm{~cm}$. La medida de longitud, en tanto convencional, no se basa en leyes físicas, sino en disposiciones observacionales, que permiten la comparación de un objeto con otro dado. Tales disposiciones son: 
1) Si se hace coincidir el inicio de dos objetos y el final de uno coincide con el final del otro, tienen igual longitud.

2) Un objeto debe ser mayor que cualquiera de sus partes propias.

Ambos preceptos también hacen posible determinar si un objeto es más largo o menos largo que otro. Pero ello depende de tres supuestos fundamentales:

3) Que siempre es posible hacer coincidir, en su inicio o en su final, dos objetos.

4) Que cualquier parte propia de un objeto está totalmente contenida en él.

5) Que cualquier objeto es divisible en cualquier número de partes propias.

Estos tres postulados son objetables.

1) Hace referencia al principio de identidad, que es problemático si se considera a la magnitud misma. Así, se podría pensar en comparar la 'longitud' de dos informaciones haciéndolas coincidir en su principio y mirando su fin, pero es dudoso que se puedan alinear perfectamente entidades que tienen fronteras borrosas, no definidas. Lo mismo ocurre con objetos físicos, como las montañas; nadie duda de que el Everest es una parte de la cordillera del Himalaya, pero cualquiera tendría serias dudas si tuviera que decidir dónde empieza.

La identidad entre dos entes ha estado tradicionalmente caracterizada por el principio de identidad de los indiscernibles, que dice que dos objetos que tienen las mismas propiedades son la misma cosa. La distinción entre propiedades esenciales y accidentales permite una debilitación de este principio: si dos objetos tienen las mismas propiedades esenciales y difieren en las accidentales serán mucho más indistinguibles que si comparten propiedades accidentales y no esenciales.

2) Refiere a la noción de subconjunto. En el sentido tradicional, un conjunto B es un subconjunto de un conjunto A si todo x que es un elemento de B también lo es de A. Así, es claro que el conjunto \{1,2,3\} está contenido en el conjunto $\{1,2, \ldots, 9,10\}$; es decir, que un objeto que mida 1,2 o $3 \mathrm{~cm}$, puede ponerse en correspondencia con una regla de $10 \mathrm{~cm}$. y medirlo contra ella porque su mensura está contenida en la de la regla. Pero hay objetos físicos donde la relación 'estar contenido en' es borrosa. Las taxonomías biológicas o las categorías gramaticales, aunque se traten de caracterizar de modo preciso, son un claro ejemplo de borderline cases, porque hay elementos fronterizos refractarios a ser separados con nitidez. Así, Ross mostró que, entre las categorías gramaticales de verbo y nombre hay un continuo que se muestra en la siguiente serie: Verbo $>$ Participio Presente $>$ Adjetivo $>$ Preposición > Nombre adjetivado > Nombre, de manera que es dudoso si la palabra 'divertido' es clasificable como 'Nombre adjetivado' o como 'Nombre’ (Ross, 2004). También en lingüística, se caracteriza al homo sapiens como homo loquens, pero es controvertido si entre la ausencia de lenguaje o su presencia plena existe un intermedio donde las palabras ya tienen significado pero apenas estructura sintáctica (Bickerton, 1990). En biología, es controvertido si los eslabones perdidos se asemejan más a los primates o a los humanos (Savage-Rumbaugh; Lewin, 1994).

3) Supone que todos los objetos son divisibles y que esas divisiones son reconocibles como tales. La cuestión de que todos los objetos constan de partes es objetable. Así, en física, las partículas elementales son aquellas que no están constituidas por entidades más pequeñas ni tienen estructura interna alguna. Dejando a un lado este caso, las partículas complejas sí tienen partes organizadas de alguna manera y a ellas nos referiremos, porque incluso reduciendo sus partes a divisiones precisas en una regla imaginaria, surgen problemas. Así, de forma observacional podemos discriminar las diferentes partes longitudinales de un objeto en una regla: eso permite emparejar el inicio del objeto a medir y el de la regla y determinar su mensura en términos de la coincidencia de su fin con una de las divisiones de la regla. Pero cada división se marca con una raya y esa raya ocupa un espacio. Usando un instrumento de observación más potente, esa separación que a simple vista parece finísima, sería groseramente gruesa y susceptible, a su vez, de ser compartimentada, consiguiendo así divisiones más finas de la regla. Pero esas particiones estarían, a su vez, delimitadas por rayas que, con un instrumento 
más afinado, serían susceptibles de seguir siendo divididas...en un proceso que, en teoría, es recursivamente ilimitado. Esto indica que la medida depende de la naturaleza del objeto -que sea mensurable-, pero también del instrumento de medida. En el mundo cuántico, esto alcanza especial relevancia, porque solo accedemos al objeto a través del instrumento y este determina la realidad del objeto en tanto posibilita nuestro acceso epistémico a él.

1.3. Al principio hablamos de las propiedades cualitativas como diferentes de las propiedades cuantitativas. Las propiedades cualitativas son observables, como las cuantitativas, pero a diferencia de ellas, a veces no admiten ser fácilmente sustituidas por números. Los objetos que comparten una propiedad cualitativa se agrupan en una categoría nominal; es decir, en un nombre o entidad lingüística y no numérica, que lo determina. En un escenario informacional, 'noticiable/de relleno/no noticiable', 'primicia/de actualidad/diario/pasado', 'segura/fiable/sospecha/rumor'” son categorías cualitativas. Estas se caracterizan por ser multidimensionales: un suceso puede ser noticiable para un periodista de una ideología, pero no para otro con una ideología diferente; puede ser noticiable para un periódico de provincias, pero no para uno nacional, etc. Esto hace que sea difícil, sino imposible, reunir de una vez por todas y de manera exhaustiva a todos los componentes que definirían a una categoría nominal, porque son contextuales y de textura abierta: es decir, evolucionan en el tiempo y son susceptibles de variar. Sus límites son borrosos. Para las propiedades cualitativas parece difícil establecer una única medida exacta y un orden preciso. En algunas ocasiones podremos decir que $a$ es mayor, menor o igual a $b$, pero en otros sólo podremos estimar que $a$ parece mayor o menor que $b$ o que $a$ y $b$ son indistinguibles.

Las modernas tecnologías de la información usan medidas para cuantificar, p. ej., la bondad de los buscadores en Internet. Estas medidas se basan fundamentalmente en dos acciones: las páginas recuperadas y la relevancia de las mismas. Lo que se recupera es una propiedad cuantitativa representable con un número. Ante una búsqueda con una palabra clave, el buscador debe rescatar el mayor número de páginas que incluyan a esa palabra. Como suelen ser muchas, el criterio verdaderamente importante es la relevancia: dar prioridad a aquellas que, incluyendo el término de la búsqueda, proporcionen datos importantes acerca de la misma. Y la relevancia es un criterio esencialmente cualitativo. Resulta de vincular un objetivo y un contexto (buscar información de 'banco' en la acepción 'mobiliario' y referido a parques es un asunto de grado (los parques pueden tener calles; por tanto, no es totalmente, pero si parcialmente relevante, si el buscador ofrece información de bancos que usualmente se instalan en las calles) y puede ser representada por juicios comparativos (la información sobre bancos que se instalan en las calles es más relevante que la de bancos que se instalan en los hospitales).

Para estos casos de medidas que son no aditivas (la consulta de la suma de muchas páginas no relevantes no supone obtener una información relevante); de medidas no soportadas por propiedades físicas gobernadas por leyes, sino por convenciones; de medidas que soportan mejor un orden que la adscripción precisa de un número (esta página es relevante/irrelevante o es relevante con grado 0,7 frente a esta página es más relevante que esta otra -según el contexto y para el objetivo señalado-), sugiere la necesidad de nuevos instrumentos matemáticos que ayuden a una representación fiel de las cualidades de los objetos. Fidelidad no quiere decir precisión imaginaria, sino adecuación, con la exactitud posible, a la realidad conocida.

1.4. En la descripción del mundo, a veces se antepone la razón a la realidad. De las llamadas 'leyes de la razón' se dice que muestran la naturaleza de las cosas. Por ejemplo: cualquier objeto tiene una propiedad o su contraria y nada en el medio según el principio del tercero excluido. Para los defensores de la supremacía de la 'razón precisa', el motivo de que los objetos no se acomoden a esos preceptos se debe al conocimiento deficiente de los mismos, pero no a su verdadero ser, inmutable e independiente de contexto y propósito. Con esta visión platónica se regimenta al lenguaje que expresa al mundo y se hace casar con las leyes de la razón. Pero el avance del conocimiento surge de examinar la realidad sin prejuicios, como se da a nuestros sentidos y conocimiento, y de hallar quizás otros preceptos de la razón que expliquen mejor los objetos y las cosas no prejuzgadas. La noción de 
magnitud escalar en ámbitos como las ciencias de la información parece demandar esos nuevos principios.

\section{2. ¿Cuál es el problema?}

Nuestros estudiantes siguen recibiendo la misma formación de hace más de setenta años por lo que respecta al concepto de magnitud escalar, entonces basado en ideas provenientes de entidades medibles del mundo macro-físico como longitudes, áreas y volúmenes (Arregui, 1969). De ellas y razonablemente, puede suponerse que no sólo pueden representarse en retículos, sino que 'crecen' por agregación de partes disjuntas; son entidades que cabe entender como un conjunto de 'partes separadas', como son, también, los sucesos aleatorios del tipo 'lanzar un dado y apuntar el número de puntos que salen en cada tirada', los cuales se representan bien en un álgebra de Boole (Birkhoff, 1940), el tipo de retículo dotado del mayor número posible de propiedades algebraicas y en el cual es a $+b=a+a^{\prime} \cdot b$, ya que la ley distributiva permite el cálculo $a+a^{\prime} \cdot b=\left(a+a^{\prime}\right) \cdot(a+b)=a+b$, con $a \cdot$ $(a ’ b)=0$. Los axiomas que definen un álgebra de Boole fuerzan que toda unión pueda expresarse como una unión de partes disjuntas, algo que falla tan pronto algunas de esas leyes dejan de ser válidas; es el caso del razonamiento con la multitud de conceptos imprecisos que permean el lenguaje ordinario, que no puede modelarse en un álgebra de Boole, como sucede cuando el razonamiento sólo concierne palabras precisas y como es también el caso del razonamiento de la física cuántica, para el cual y sin embargo no valen las leyes distributivas.

Con todo y como se ha dicho en la introducción, las actuales ciencias de la información y las comunicaciones requieren una ampliación de aquel concepto de magnitud escalar para comprender los nuevos objetos que se han ido introduciendo, estudiando y midiendo por lo menos a lo largo del último medio siglo (De Luca; Termini, 1972).

2.1. En cierta forma, como también se apuntó en la introducción, ello provenía de la suposición macroscópica de que el 'todo' considerado coincide con una unión (disjunta) de 'sus partes', lo cual presupone, en primer lugar, que cabe reconocer y distinguir las partes que componen el correspondiente todo; que los 'todos' crecen por yuxtaposición de partes. Sin embargo, a lo largo de esos años han aparecido nuevas ideas y estudios respecto de entidades diferentes, como son las permeadas de imprecisión o de vaguedad y que, al poder ser medidas, también se constituyen en magnitudes para las cuales, no obstante, ni cabe siempre suponer que sus elementos pueden representarse en un retículo, ni que 'crecen’ de manera disjunta (Trillas, 2017). Con ello, pueden crecer sin yuxtaposición en forma no aditiva y no como, por ejemplo, lo hacen longitudes, áreas, volúmenes, sucesos aleatorios y algunos conceptos físicos. Para abarcar determinados temas de las actuales ciencias y tecnologías de la información se requiere revisar el antiguo concepto de magnitud escalar de forma que, sin invalidarlo, se amplíe convenientemente; se trata de temas que siendo ya de gran importancia económica y social, no deberían dejar de tenerse en cuenta en la formación matemática.

El antiguo concepto se basa, por decirlo llanamente, en una especie de 'principio de no penetrabilidad' de los cuerpos, pero actualmente hay que abrirlo a entidades nebulosas de las que con frecuencia es difícil, sino imposible, saber dónde empiezan, dónde acaban y de qué partes constan; metafóricamente, son entidades cuyas partes no son nítidas, se penetran de manera filamentosa y no es siempre verosímil suponer que forman un retículo. Con ello y por ejemplo, el símil de la intersección de conjuntos representando la conjunción copulativa ' $y$ ' del lenguaje ordinario no puede llevarse más allá de las palabras precisas; ni siempre la conjunción lingüística puede asimilarse a la operación conjunción reticular, ni puede suponerse que es conmutativa o asociativa como lo sería necesariamente de siempre poderse representar en un retículo (Rey Pastor, 1941).

Conviene recordar que, aún más antiguamente, el concepto de magnitud se refirió simplemente a aquello que crece o decrece y a lo que puede asignársele números que varían en forma concomitante (Trillas, 2017); por ejemplo, el suceso 'obtener tres puntos' al tirar un dado puede crecer hasta el 
suceso 'obtener un número impar de puntos' por yuxtaposición de partes en forma secuencialmente disjunta:

'obtener tres', 'obtener uno’, 'obtener cinco', hasta 'obtener tres, uno y cinco’,

que corresponde a 'obtener una puntuación impar' entre los posibles resultados $\Omega=\{1,2,3,4,5,6\}$ obtenibles al lanzar un dado. El crecimiento de esos sucesos aleatorios puede representarse por medio de la relación de inclusión $(\subseteq$ ) entre los conjuntos que los representan (por ejemplo, es $\{3\} \subseteq\{1,3,5\}$ ) y el par formado por las representaciones conjuntistas de todos los resultados posibles de un experimento aleatorio y la relación de inclusión es una magnitud que, una vez se le añade una cantidad, la probabilidad, deviene una magnitud escalar medida en el semi-grupo conmutativo totalmente ordenado $S=([0,1], \diamond$; $\leq)$; es decir, en el intervalo unidad de la recta real dotado de la operación 'suma acotada', $\mathrm{a} \diamond \mathrm{b}=\min (1, \mathrm{a}+\mathrm{b})$. La correspondiente magnitud escalar viene mostrada por la terna $((\Omega, \subseteq)$, S, prob), siendo prob: $X \rightarrow[0,1]$ la probabilidad elegida para 'medir' sucesos aleatorios, como sucede, por ejemplo, con $\mathrm{A}=$ 'obtener un número impar' de puntos que, de acuerdo con la definición de probabilidad, es prob $(A)=\operatorname{prob}(\{1\}) \diamond \operatorname{prob}(\{3\}) \diamond \operatorname{prob}(\{5\})=\operatorname{prob}(\{1\})+$ prob $(\{3\})+\operatorname{prob}(\{5\})$, ya que esa suma acotada a lo sumo vale 1 . Con ello, es obvio que si los sucesos crecen según la relación de inclusión, sus medidas (probabilidades) también crecen según el orden de la recta real; así, si $\mathrm{B}=\{1,3\}$, es $\mathrm{B} \subseteq \mathrm{A}$, y prob $(\mathrm{B})=\operatorname{prob}(\{1\}) \diamond \operatorname{prob}(\{3\})=\operatorname{prob}(\{1\})+$ prob $(\{3\}) \leq$ prob (A). Nótese que la probabilidad es aditiva por definición; no sólo la probabilidad está sometida al principio de que la probabilidad de $\Omega$ debe ser 1 , sino que si $\mathrm{A} \cap \mathrm{B}=\varnothing$, es prob ( $\mathrm{A} \cap$ B) $=$ prob (A)+prob (B).

De la misma manera, el conjunto de todos los segmentos rectilíneos en un plano euclidiano, con la relación 'menor que' establecida entre ellos por 'transporte geométrico', es una magnitud que deviene escalar al añadirle la longitud medida en el semi-grupo conmutativo y totalmente ordenado $\left(\mathrm{R}^{+}, \leq ;+\right)$, de la semirrecta real cerrada en un extremo y abierta en el otro, el intervalo $[0,+\infty)$. Lo mismo sucede con los cuadrados en el plano y los cubos en el espacio; en todos ellos vale la ley aditiva (Abellanas, 1963.

2.2. Así, el mínimo esencial para contar con una magnitud escalar es disponer de un conjunto $\Omega$ de objetos dotado de una relación binaria $\rightarrow(\subseteq \Omega \times \Omega)$ entre ellos y de una aplicación m que traslade estos objetos a los valores en un conjunto $\mathrm{V}$ dotado de una estructura ordenada por otra relación $\nearrow(\nearrow$ $\subseteq \mathrm{V} x \mathrm{~V}), \mathrm{m}: \Omega \rightarrow \mathrm{V}$ ), de tal manera que,

$$
\text { Cada vez que sea } \mathrm{A} \rightarrow \mathrm{B} \text {, se siga } \mathrm{m}(\mathrm{A}) \nearrow \mathrm{m}(\mathrm{B}) \text {. }
$$

Cuando V es un conjunto de números, la magnitud $(\Omega ; \rightarrow)$ deviene escalar tan pronto se fija el par (V, $\nearrow)$ y se agrega la aplicación $\mathrm{m}: \Omega \rightarrow \mathrm{V}$; cabe sintetizarla en la terna $((\Omega ; \rightarrow) ;(\mathrm{V}, \nearrow) ; \mathrm{m})$.

El antiguo concepto a ampliar proviene de suponer que $\Omega$ es un conjunto de partes (subconjuntos) de un universo, que $\rightarrow$ es la relación de inclusión $(\subseteq)$ entre subconjuntos y que $\nearrow$ es bien el orden total de la recta real, bien el orden parcial de los números complejos, como es el caso de ciertas magnitudes eléctricas (Menger, 2007). Es decir, que V es, bien el conjunto de los números reales, bien el de los números complejos. En ese concepto antiguo se supone que la medida crece en forma aditiva; es decir, que si A está en $\Omega$ y se descompone en partes $A_{1}$ y $A_{2}$ disjuntas $\left(A=A_{1} \cup A_{2}\right.$, tales que $\left.A_{1} \cap A_{2}=\varnothing\right)$, entonces es $m(A)=m\left(A_{1}\right)+m\left(A_{2}\right)$.

Sin embargo, actualmente se recurre también a medidas de conjuntos clásicos que no son aditivas y como son, por ejemplo, las medidas-lambda (Alsina, et.al, 2006) $\left(\mathrm{m}_{\lambda}: 2^{\Omega} \rightarrow[0,1]\right.$, para $\left.\lambda>-1\right)$, definidas por las propiedades

$$
\text { 1) } \mathrm{m}_{\lambda}(\Omega)=1, \mathrm{y}
$$


2) $\mathrm{m}_{\lambda}(\mathrm{A} \cup \mathrm{B})=\mathrm{m}_{\lambda}(\mathrm{A})+\mathrm{m}_{\lambda}(\mathrm{B})+\lambda \mathrm{m}_{\lambda}(\mathrm{A}) \mathrm{m}_{\lambda}(\mathrm{B})$, si $\mathrm{A} \cap \mathrm{B}=\varnothing$.

Obsérvese que con $B=A^{\prime}$, resulta $m_{\lambda}\left(A A^{\prime}\right)=m_{\lambda}(\Omega)=1=m_{\lambda}(A)+m_{\lambda}\left(A^{\prime}\right)\left(1+\lambda m_{\lambda}(A)\right)$, con lo cual es $\mathrm{m}_{\lambda}\left(\mathrm{A}^{\prime}\right)=\left(1-\mathrm{m}_{\lambda}(\mathrm{A})\right) /\left(1+\lambda \mathrm{m}_{\lambda}(\mathrm{A})\right) \mathrm{y}$, en particular, $\mathrm{m}_{\lambda}(\varnothing)=\mathrm{m}_{\lambda}\left(\Omega^{\prime}\right)=0$.

Es obvio que esas medidas sólo son aditivas para $\lambda=0$, en tanto que si $-1<\lambda<0$ son sub-aditivas y si $\lambda>0$ son super-aditivas; es decir, la medida de un 'todo' es, respectivamente menor o mayor que la suma de las medidas de las partes y sólo en el caso aditivo la medida del todo coincide con la suma de las medidas de las partes. Está claro que, incluso si no es $\lambda=0$, también $\left((\Omega, \subseteq), \mathrm{m}_{\lambda}\right)$ merece ser considerada una magnitud escalar y lo mismo sucede si m es, bien una medida de posibilidad, bien una de necesidad, ambas tampoco aditivas (Trillas; Eciolaza, 2015). Por ejemplo, las propiedades esenciales de una medida de posibilidad son $\mathrm{m}(\Omega)=1, \mathrm{~m}(\varnothing)=0$ y $\mathrm{m}(\mathrm{A} \cup \mathrm{B})=\max (\mathrm{m}(\mathrm{A}), \mathrm{m}(\mathrm{B})$ ), cualesquiera que sean $\mathrm{A}$ y $\mathrm{B}$, tanto si son disjuntos como si no lo son; en ambos casos, es $\mathrm{m}(\mathrm{A} \cup \mathrm{B}) \leq$ m (A) + m (B) y, por lo tanto, las medidas de posibilidad son sub-aditivas.

\section{Notas}

1) En el caso de la llamada lógica de la física cuántica, en la cual no valen las leyes distributivas ni la ley del reparto perfecto, según la cual para cualesquiera a y b en un álgebra de Boole es $a=a \cdot b+a$ $\cdot$.b' (a se reparte perfectamente entre b y su complemento b'), ya que es a $=a \cdot 1=a \cdot\left(b+b^{\prime}\right)=a \cdot b+$ $\mathrm{a} \cdot \mathrm{b}$ ', tampoco vale la equivalencia booleana entre incompatibilidad $(\mathrm{a} \cdot \mathrm{b}=0)$ y contradicción $(\mathrm{a} \leq$ b’). En las álgebras de Boole, esta equivalencia se deduce de la manera siguiente:

$$
\begin{aligned}
& \text { I: Si es } a \leq b^{\prime} \text {, sigue } a \cdot b \leq b^{\prime} \cdot b=0 \text {, que implica } a \cdot b=0 \text {; } \\
& \text { II: Si es } a \cdot b=0 \text {, de } a=a \cdot b+a \cdot b^{\prime}=a \cdot b^{\prime} \text { se sigue } a \leq b \text {. }
\end{aligned}
$$

Como consecuencia de esa falta de equivalencia, el crecimiento puede contemplarse de dos maneras diferentes (Alsina, C; E. Trillas, Ll. Valverde, 1983): $\mathrm{Si} a=\mathrm{a}_{1}+\mathrm{a}_{2}$, tanto puede ser que $\mathrm{a}_{1} \cdot \mathrm{a}_{2}=0$ (unión disjunta), como $\mathrm{a}_{1} \leq \mathrm{a}_{2}$ ' (unión contradictoria). Obsérvese que, al ser válida la prueba I en cualquier orto - retículo, la contradicción implica la incompatibilidad en todos ellos, pero que al no valer siempre la prueba II, en general la incompatibilidad no implica contradicción; así, en la lógica cuántica existen elementos incompatibles que no son contradictorios. En el lenguaje ambas propiedades son, en general, independientes (Trillas, 2017).

2) Que, en las álgebras de Boole, la probabilidad sea una función creciente es consecuencia de su ley aditiva y gracias a que es $A \subseteq B \Leftrightarrow B=A \cup B=A \cup\left(A^{\prime} \cap B\right)$, una unión disjunta de la que se sigue que prob $(B)=\operatorname{prob}(A)+\operatorname{prob}\left(A^{\prime} \cap B\right)>\operatorname{prob}(A)$.

3) La definición antigua y más usual entre los matemáticos de magnitud escalar es aquella según la cual la magnitud es un semigrupo aditivo abeliano con una ordenación arquimediana y cuyos elementos se llaman cantidades (Arregui, 1969). Es una definición un tanto abusiva ya que permite confundir los elementos a medir con sus medidas; por ejemplo, en el caso de sucesos aleatorios permitiría confundir el suceso A con su prob (A) y sin que esa correspondencia sea biyectiva ya que, por ejemplo, tanto el suceso 'pares' $\{2,4,6\}$, como el 'impares' $\{1,3,5\}$ tienen la misma probabilidad $1 / 2$ con tal de que la probabilidad de cada suceso elemental sea $1 / 6$.

Conviene, en este punto, recordar que las t-normas y las t-conormas continuas, empleadas en la lógica borrosa teórica para representar, respectivamente, los conectivos lingüísticos ' $y$ ', y 'o', son precisamente operaciones conmutativas que dotan al intervalo $[0,1]$ de la estructura de semigrupo abeliano y algunos de los cuales son arquimedianos (Trillas, 2017; Trillas; Eciolaza, 2015); precisamente, como tales semigrupos fueron introducidas en la lógica borrosa (Alsina, Trillas, Valverde, 1983). De ellas, ni la t-norma min, ni las sumas ordinales son arquimedianas, siéndolo las que pertenecen a las familias del producto y de Lukasiewicz (Trillas; Eciolaza, 2015; Alsina, Frank, 
Schweizer, 2006); algo que refleja la no generalidad del carácter arquimediano en las cantidades de las nuevas magnitudes.

4) Una operación * entre números reales es arquimediana si dados $x<y$, con $x>0$, existe un número entero $\mathrm{n}>0$ tal que es $\mathrm{y}<\mathrm{n} * \mathrm{x}$. Obviamente, el producto de números es una operación arquimediana con la que cabe establecer la propiedad en la forma $\mathrm{y} / \mathrm{n}<\mathrm{x}$, permitiendo conjeturar que siempre hay una 'parte' de la magnitud mayor que es, a su vez, parte de la menor. El mínimo de números no es, sin embargo, una operación arquimediana; en efecto, de ser $\mathrm{y}<\min (\mathrm{n}, \mathrm{x})$ al no poder ser $\mathrm{y}<\mathrm{x}$, no puede ser $\min (\mathrm{n}, \mathrm{x})=\mathrm{x}$ (equivalente a $\mathrm{x}<\mathrm{n})$, sino que debe ser $\min (\mathrm{n}, \mathrm{x})=\mathrm{n}$, que lleva a $\mathrm{y}<\mathrm{n}$ con lo que también resultaría $\mathrm{x}<\mathrm{n}$. Por lo que respecta a la t-norma de Lukasiewicz, $\mathrm{W}(\mathrm{a}, \mathrm{b})=\max (0, \mathrm{a}+\mathrm{b}-$ $1)$, ¿es $\mathrm{y}<\mathrm{W}(\mathrm{n}, \mathrm{x})=\max (0, \mathrm{n}+\mathrm{x}-1)$ ? Ello es equivalente a $\mathrm{y}<\mathrm{n}+\mathrm{x}-1 \Leftrightarrow \mathrm{y}+1<\mathrm{n}+\mathrm{x}$, evidentemente verificado por algún entero positivo $n$. Es decir, la operación $\mathrm{W}$ es arquimediana.

En conclusión, en la composición de las cantidades de una magnitud (con valores reales) por medio de una operación arquimediana, cabe suponer que (entre esas magnitudes) toda magnitud mayor tiene una parte incluida en la magnitud menor. Sin embargo, cuando no se dé tal característica de las magnitudes, no parece necesario emplear una operación arquimediana; ello sucede de tomar $*=\min$, operación que es frecuentemente empleada en las aplicaciones de la lógica borrosa.

\section{Un nuevo concepto de magnitud escalar}

3.1. Para definir una magnitud escalar es necesario, en primer lugar, contar con un conjunto de elementos $\Omega=\{\mathrm{a}, \mathrm{b}, \mathrm{c}, \ldots\}$ dotado de una relación binaria $<\subseteq \Omega \times \Omega$, tal que con $\mathrm{a}<\mathrm{b}$ se indique 'a es menos que b' con referencia a mostrar alguna propiedad o propiedades reconocibles en y entre los elementos de $\Omega$. El par $(\Omega,<)$ es una magnitud. En segundo lugar y para llegar al concepto de magnitud escalar, es necesario un acuerdo respecto de qué significa medir los objetos de la magnitud $(\Omega,<)$ (Menger, 2007).

Tal acuerdo sólo puede provenir de lo que refleje la relación binaria < . Sí, tal como parece, que 'a sea menos que b’ $(\mathrm{a}<\mathrm{b})$ refleja, en cierto sentido, el crecimiento de lo que se reconoce en y entre los elementos de $\Omega$, entonces y entendiendo el medir como una asignación a cada elemento de $\Omega$ de un 'valor' (a $\rightarrow$ m (a)) que crezca al crecer a, habrá que convenir dónde encontrar esos valores; habrá que fijar su conjunto al igual que, con las probabilidades, se fija el intervalo unidad, al provenir el concepto de probabilidad del cociente entre el número de sucesos favorables y el número total de sucesos. Naturalmente, ese conjunto de valores deberá estar provisto de una relación de orden $(\leq)$ que permita pasar desde $\mathrm{a}<\mathrm{b}$, hasta $\mathrm{m}(\mathrm{a}) \leq \mathrm{m}(\mathrm{b})$; si a es menos que $\mathrm{b}$, entonces a mide menos que $\mathrm{b}$.

Debe observarse que el orden $\leq$ no tiene por qué ser, necesariamente, total o lineal, como lo es el orden de los números reales; por ejemplo, el orden entre los números complejos es sólo parcial ya que existen pares de números complejos que no son comparables entre ellos en el orden usual definido por:

$$
\mathrm{a}+\mathrm{ib} \leq \mathrm{c}+\mathrm{id} \Leftrightarrow \mathrm{a} \leq \mathrm{c} \mathrm{y} \leq \mathrm{d},
$$

siendo el segundo signo $\leq$ el orden total de la recta real. Por ejemplo, los números complejos $2+5 i$ y 3 - $4 \mathrm{i}$ no son comparables, ya que ni es $2+5 \mathrm{i} \leq 3-4 \mathrm{i}$, ni es $3-4 \mathrm{i} \leq 2+5 \mathrm{i}$, al ser $3>2$ pero $-4<5$. Así, en el campo de la física la impedancia eléctrica se mide con números complejos.

Nota.

Si en lugar de considerar los números complejos a + ib, se considerasen los vectores de coordenadas cartesianas $(\mathrm{a}, \mathrm{b})$, entonces cabría decir que la magnitud $(\Omega,<)$ es una magnitud 'vectorial' en lugar de escalar (Menger, 2007); sin embargo y en tal caso, las operaciones con números complejos deberán cambiarse por las de vectores en el plano $\mathrm{R}^{2}$. Las magnitudes vectoriales asignan no sólo un número, la norma $\left(a^{2}+b^{2}\right)^{1 / 2}$ del vector $(a, b)$, sino también un sentido y una dirección dadas por el 
correspondiente vector; con sólo la norma, se tendría otra magnitud escalar y no necesariamente aditiva. Entre las magnitudes vectoriales, típicas de la física, están la velocidad, la aceleración y el campo eléctrico.

3.2. Usualmente, el concepto de medida asignada a una magnitud $(\Omega,<)$, requiere fijar una 'unidad' de medida; para ello, basta considerar los elementos de $\Omega$, tales que ningún otro elemento es 'más' que ellos, si existen. Es decir, a es maximal respecto de la relación $<$, supuesto que no existe ningún $\mathrm{x}$, distinto de a, tal que sea $\mathrm{a}<\mathrm{x}$; i.e., que el conjunto $\{\mathrm{x} \in \Omega$; $\mathrm{a}<\mathrm{x}\}$ sea vacío. Análogamente, un elemento b es minimal si ninguno es 'menos' que él; es decir, $\{\mathrm{y} \in \Omega ; \mathrm{y}<\mathrm{b}\}$ es vacío, supuesto que y es distinto de b. De existir un único maximal se llama el máximo y de existir un único minimal, el mínimo.

Con ello, si el conjunto $\mathrm{V}$ de los valores, ordenado por la relación $\leq$, contiene un máximo (maximal único) 1 y un mínimo (minimal único) 0, los tres axiomas que definen una medida son:

1) Si $\mathrm{a}<\mathrm{b}$, entonces $\mathrm{m}(\mathrm{a}) \leq \mathrm{m}(\mathrm{b})$;

2) Si a es maximal, entonces $\mathrm{m}(\mathrm{a})=\mathbf{1}$;

3) Si b es minimal, entonces $\mathrm{m}(\mathrm{b})=\mathbf{0}$.

Los elementos maximales pueden verse como 'prototipos' de la magnitud y los elementos minimales como 'anti-prototipos'; ambos son importantes para ser empíricamente reconocidos. Nótese que si V es el intervalo unidad [0,1], entonces es $\mathbf{1}=1$ y $\mathbf{0}=0$; de ser $\mathrm{V}=[\mathrm{a}$, b], sería $\mathbf{0}=\mathrm{a}$ y $\mathrm{b}=\mathbf{1}$; de emplearse los números del intervalo unidad complejo, sería $\mathbf{0}=0+\mathrm{i} 0$ y $\mathbf{1}=1+\mathrm{i}$. Se trata de una definición inspirada en la de medida borrosa previamente introducida por Michio Sugeno (Sugeno, M., 1974; Trillas, 2017).

Cuando no existen maximales, cabe elegir un elemento al que asignar la medida unidad como en el caso de la longitud de segmentos, donde basta escoger uno de ellos como unidad; son los casos del metro lineal, del metro cuadrado, del metro cúbico, del suceso total, etc. De igual forma, cabe elegir un elemento al que asignar la medida cero, como los puntos de la recta para la longitud; los segmentos [a, a $]=\{a\}$, o el conjunto vacío en el caso de las probabilidades. Se trata de prototipos y anti-prototipos elegidos artificialmente para facilitar la comparación y la correspondiente medición.

Con todo ello, la magnitud $(\Omega,<)$, queda dotada de una medida o cantidad m, y cada valor m (a) es la cantidad asociada al elemento a de $\Omega$. Un ejemplo, correspondiente a la renovación del concepto de magnitud escalar, es conveniente.

En el universo de los números de la recta real entre 0 y 10, ambos incluidos, considérese el concepto G = grande. ¿Genera $G$ una magnitud escalar? Para responder a la pregunta hay que saber, en primer lugar, cuál es el significado de $\mathrm{G}$ en el intervalo cerrado $[0,10]$, es decir cuando se afirmará que de dos números x e y entre 0 y 10, uno es 'menos grande' que el otro, lo que, obviamente, sucederá si y sólo si es $\mathrm{x} \leq \mathrm{y}$, de manera que serán 'igualmente grandes' cuando $\mathrm{x}=\mathrm{y}$. Con ello, la magnitud a considerar es el par $([0,10], \leq)$ donde no hay más que un maximal 10 (el máximo) y un minimal 0 (el mínimo). Elegido como conjunto ordenado de valores para una medida el intervalo unidad $[0,1]$, se tendrá una magnitud escalar $(([0,10], \leq),([0,1], \leq), \mathrm{m})$, cada vez que se especifique una medida $\mathrm{m}:[0,10] \rightarrow[0$, 1]. Tales medidas deberán satisfacer los axiomas:

1) Si $x \leq y$, entonces $m(x) \leq m(y)$;

2) $\mathrm{m}(10)=1$;

3) $\mathrm{m}(0)=0$, 
De ellas existe la enorme multiplicidad de todas las funciones que son crecientes entre los puntos $(0,0)$ y $(10,1)$ del plano. El concepto $G$ genera en $[0,10]$ la magnitud $([0,10], \leq)$, pero no genera una única magnitud escalar sino muchas y, para especificar una de ellas, se requiere, bien más información, bien establecer algunas hipótesis razonables sobre la misma; esa información adicional y/o hipótesis razonables permitirá diseñar una medida. Así, de aceptarse que el crecimiento de m es lineal, es decir, que es $\mathrm{m}(\mathrm{x})=\mathrm{ax}+\mathrm{b}$, entonces, $\mathrm{y}$ como la derivada de la función $\mathrm{m}, \mathrm{m}$ ' $(\mathrm{x})=\mathrm{a}$, debe ser positiva, las condiciones 2 y 3 llevan inmediatamente a $b=0$ y a $=1 / 10>0$, con lo cual la medida es $\mathrm{m}(\mathrm{x})=\mathrm{x} / 10 \mathrm{y}$ se dispone de la magnitud escalar $(([0,10], \leq), \mathrm{x} / 10)$. De suponer que la medida es cuadrática, $\mathrm{m}(\mathrm{x})=$ $a x^{2}+b x+c$, de (3) sigue $c=0$, de (2) $1=100 a+10 b$, debiendo ser, además, positiva su derivada m’ $(\mathrm{x})=2 \mathrm{ax}+\mathrm{b}$ en todos los puntos $\mathrm{x} \in[0,10]$; está claro que para especificar una medida cuadrática se requiere alguna condición o hipótesis más. Así, de poderse conocer que la curva descrita por la función $\mathrm{m}$ debe pasar por el punto $(5,0.7)$, es decir, que es $\mathrm{m}(5)=25 a+5 b=0.7$, se dispondrá de dos ecuaciones lineales en las dos incógnitas a y b que permiten calcularlas fácilmente, resultando $\mathrm{a}=$ $0.4 / 50=-0.008, \mathrm{~b}=1.8 / 10=0.18$. La correspondiente medida cuadrática es, por tanto, $\mathrm{m}(\mathrm{x})=-$ $0.008 x^{2}+0.18 x$, que, al ser $-0.016 x+0.18>0(\Leftrightarrow x<11.25)$ para todo $x \in[0,10]$, es efectivamente creciente. La magnitud generada por $\mathrm{G}$ en el intervalo $[0,10]$ no tiene una medida única, sino un conjunto de ellas que, no obstante, siguen unas reglas comunes; con ello, no son únicas las cantidades numéricas asignadas a cada elemento de $[0,10]$.

Nótese que la suposición de que m es derivable implica su continuidad; sin embargo, cabe obviar esta suposición y especificar medidas no continuas, como es, por ejemplo, $\mathrm{m}(\mathrm{x})=0$ si $0 \leq \mathrm{x} \leq 7, \mathrm{y} \mathrm{m}(\mathrm{x})=$ 1 si $7<\mathrm{x} \leq 10$, que interpreta el (impreciso) concepto 'grande' como el concepto (preciso) 'mayor que 7'; hay los prototipos en el intervalo $(7,10]$ y los anti-prototipos en el intervalo [0, 7] y la medida tiene un 'salto' en el entorno (derecho) de $\mathrm{x}=7$. De forma análoga, pueden definirse medidas discontinuas con un número finito de valores; por ejemplo, con tres:

$$
\begin{aligned}
& \mathrm{m}(\mathrm{x})=0, \text { si } 0 \leq \mathrm{x}<3 ; \\
& \mathrm{m}(\mathrm{x})=0.5, \text { si } 3 \leq \mathrm{x}<6 ; \\
& \mathrm{m}(\mathrm{x})=1, \text { si } 6 \leq \mathrm{x} \leq 10,
\end{aligned}
$$

que supone considerar como prototipos de $\mathrm{G}$ = grande a los números en el intervalo [6,10], como antiprototipos aquellos en $[0,3)$ y como (imprecisamente) grandes con grado 0.5 a aquellos en el intervalo $[3,6)$. En este caso tri-valuado, la medida tiene dos saltos (por la izquierda) en los puntos $\mathrm{x}=3 \mathrm{y} \mathrm{x}=6$.

3.3. Una vez conocida la magnitud $(\Omega,<)$, los elementos que pueden 'identificarse bajo la relación $<$ ' son aquellos que son, a la vez, menos y más los unos que los otros; es decir, $\mathrm{a}=* \mathrm{~b} \Leftrightarrow \mathrm{a}<\mathrm{b}$ y $\mathrm{b}<\mathrm{a}$, es la relación de identificación. Todas las medidas preservan esta identificación (Alsina, Trillas, Valverde, 1983) puesto que:

$$
a=* b=>m(a) \leq m(b) \& m(b) \leq m(a) \Leftrightarrow m(a)=m(b) .
$$

En general la relación $=^{*}$ no es una equivalencia en $\Omega$, pero supuesto que $<$ fuese un pre-orden (i.e., verificase las leyes reflexiva y transitiva), entonces ${ }^{*}$ sería una relación de equivalencia (i.e., verificaría las leyes reflexiva, simétrica y transitiva), y el conjunto cociente $\Omega /=^{*}$ existiría y constaría de clases de elementos de igual medida y en número igual al de los valores distintos que efectivamente tomase $\mathrm{m}$.

En todo caso, el diseñador de la medida m no siempre puede conocer todos los pares de la relación $<\subseteq$ $\Omega \times \Omega$. Por ello y una vez haya conocido lo mejor posible tanto los elementos de $\Omega$, como la relación que hay entre ellos, diseñada una medida $\mathrm{m}$, podrá tender a confundir $<$ con la nueva relación $<_{\mathrm{m}}$ definida en $\Omega$ por (Howe; Nunes; Bryant; 2010):

$$
\mathrm{a}<_{\mathrm{m}} \mathrm{b} \Leftrightarrow \mathrm{m}(\mathrm{a}) \leq \mathrm{m}(\mathrm{b}),
$$


la cual y sin embargo, es una relación lineal o total ya que, siempre, las medidas de un par de elementos (en la recta real) serán una mayor que la otra, lo cual no sucede generalmente con < para la que fácilmente pueden existir pares de elementos incomparables, es decir, a y b tales que no sea $\mathrm{a}<\mathrm{b}$, ni sea $\mathrm{b}<\mathrm{a}$. No obstante, del hecho que $\mathrm{a}<\mathrm{b}$ implica $\mathrm{m}(\mathrm{a}) \leq \mathrm{m}(\mathrm{b})$, se sigue $\mathrm{a}<_{\mathrm{m}} \mathrm{b}$, es decir, siempre es $<\subseteq<_{\text {m }}$, la nueva relación $<_{\text {m }}$ amplía la antigua $<$; medir aumenta el significado de la magnitud tal como se vio en el ejemplo de 'grande' al tener que añadir más información o hipótesis razonables acerca del comportamiento de la función m para lograr especificarla. Se tiene así y 'a posteriori' una nueva magnitud $\left(\Omega,<_{\mathrm{m}}\right)$ que no siempre puede confundirse con la original $(\Omega,<)$, salvo que se dé la coincidencia $<=<_{\text {m, }}$ como sucede en el caso anterior de 'grande' en el cual una medida continua describe perfectamente su significado, en tanto que una discontinua lo constriñe.

$\mathrm{Si}<$ es un pre-orden, la coincidencia se da con la nueva magnitud-cociente $\left(\Omega /=*,<^{*}\right)$ constituida por las clases de equivalencia [a] $=\{\mathrm{x} \in \Omega ; \mathrm{x}=* \mathrm{a}\}$, pero ello no es general en el nuevo modelo de magnitud escalar presentado. En este modelo y contrariamente al antiguo no se definen operaciones directamente entre las (no siempre existentes) disjuntas clases de equivalencia (Arregui, 1969). No obstante, el nuevo modelo contiene al antiguo como un caso particular.

Nótese que de tomar como valores de $\mathrm{m}$ a los números complejos en lugar de los reales, entonces la relación $<_{m}$ dejaría de ser lineal; en algunas situaciones ello puede permitir una mejor aproximación entre las relaciones $<\mathrm{y}<_{\mathrm{m}}$. Análogamente sucedería de tomar los valores de la medida en cualquier otro conjunto parcialmente ordenado y, con ello se abre la posibilidad de considerar magnitudes con cantidades de tipo no directamente numérico sino, por ejemplo, funcional. Es el caso de que la información venga dada por los llamados 'números borrosos', como son, por ejemplo, 'grande' y 'entre 0.4 y 0.6 ' que se representan en $[0,1]$ por medidas mgrande $\mathrm{y} \mathrm{m}_{[0.4,0.6]}$, respectivamente, y que pueden dar lugar a valorar la medida en un subconjunto de las funciones $[0,1]^{\mathrm{X}}$.

Debe advertirse, sin embargo, que 'medir' con números borrosos (fuzzy numbers) como valores de las medidas presenta algunas dificultades, como son, por ejemplo, que el 'orden puntual' entre ellos no siempre permite reflejar bien una interpretación adecuada de esos 'números' a un problema dado, así como algún inconveniente con su 'adición' y que, por otra parte, se hace difícil establecer un número borroso mínimo y otro máximo. Tal vez, de considerar la estructura de retículo distributivo (Trillas; Eciolaza, 2015) proveniente de observar, entre los números reales de base, las operaciones 'mínimo' y ‘máximo' y extenderlas a los números borrosos mediante el ‘principio de extensión’ de Zadeh, sería posible obviar aquella dificultad para obtener magnitudes del tipo aquí propuesto y por más que la cuestión de la adecuación del orden se mantendría en pié y aparecería la dificultad de cálculo que implica el principio de extensión.

\section{Conclusión}

4.1. En el modelo antiguo se partía de suponer que la comparación $(<)$ entre los elementos de $\Omega$ era, por lo menos, un pre-orden, con lo cual se disponía automáticamente del cociente de clases disjuntas $\Omega /=*$, y las medidas podían establecerse directamente (una vez fijada una clase unidad), obedeciendo a operaciones entre las mismas clases que, usualmente, conducían a un isomorfismo con el cuerpo arquimediano de los números reales. Sin embargo, en el nuevo modelo no se requiere más allá de que $<$ sea más que una relación reflexiva para garantizar que las 'clases' módulo ${ }^{*}$ no sean vacías y $\sin$ que ello permita garantizar que esas 'clases' sean disjuntas, que formen una partición de $\Omega$; en general, $\Omega /=*$ no dará más allá de un 'recubrimiento' de $\Omega$.

El ejemplo de $G=$ grande en $[0,10]$ permite observar que sus medidas no son sino las funciones de pertenencia del subconjunto borroso en $[0,10]$ de etiqueta lingüística $G$ (Alsina, Trillas, Valverde, 1983) y que describen sus posibles estados contextuales. Por ello y en general, cada etiqueta lingüística imprecisa $\mathrm{P}$ en un universo del discurso $\mathrm{X}$ genera un subconjunto borroso de $\mathrm{X}$ que produce una magnitud escalar cada vez que se especifica una función de pertenencia (Trillas; Castro, 1995); si P es imprecisa, entonces define una única magnitud básica, pero una multitud de magnitudes escalares 
sobre aquella y cada una facilitando distintas cantidades numéricas que reflejan los estados contextuales en los que cabe considerar al conjunto borroso. Sin embargo, un subconjunto clásico de $\mathrm{X}$, completamente especificado por una etiqueta lingüística precisa, también genera una magnitud que no obstante sólo produce una única magnitud escalar. Cada terna $(X ; P ; m)$, en la que $\mathrm{m}: \mathrm{X} \rightarrow[0,1]$ es una medida o función de pertenencia al subconjunto borroso generado por la palabra $\mathrm{P}$ en $\mathrm{X}$ puede verse como una magnitud escalar; algo que no era posible con la definición antigua. Cabe decir que los subconjuntos borrosos de un universo del discurso son magnitudes escalares cuyas medidas especifican los diversos estados en los que pueden presentarse y que los subconjuntos clásicos no son sino subconjuntos borrosos/magnitudes escalares degeneradas. Así, en el conjunto de los habitantes de Madrid, la etiqueta $\mathrm{P}$ = joven, genera una magnitud tan pronto pueda reconocerse cuando un madrileño es menos joven que otro; esa magnitud no es sino el conjunto borroso ‘jóvenes madrileños' y, con ello, cada manera de medir el 'grado de juventud' llevará a una magnitud escalar, a un estado de tal conjunto borroso. Una etiqueta precisa, como es 'menor de 30 años', lleva, no obstante a una única magnitud escalar definiendo el subconjunto clásico de 'madrileños que no sobrepasan treinta años'.

4.2. Para el concepto de vaguedad es muy difícil, en general, establecer si 'esto es menos vago que aquello', lo que implica una gran dificultad para domesticar científicamente el concepto. Sin embargo, y con relación a palabras imprecisas representables por una función de pertenencia al conjunto borroso que generan, Aldo de Luca y Settimo Termini (1972) introdujeron una vía para tal domesticación en forma de la magnitud escalar generada por la palabra B = borroso que, sin duda, es un aspecto de la vaguedad o, tal vez mejor, de la imprecisión lingüística.

Supongamos que $\mathrm{P}$ genera la magnitud escalar $\left(\mathrm{X},<_{\mathrm{P}}, \mathrm{m}_{\mathrm{P}}\right)$. Los enunciados del tipo 'P es borrosa', con la palabra $\mathrm{P}$ conocida por una medida/función de pertenencia $\mathrm{m}_{\mathrm{P}}$, pueden representarse por medio de la composición de funciones $\mathrm{m}_{\mathrm{B}}$ o $\mathrm{m}_{\mathrm{P}}$ aplicada a los elementos del correspondiente universo $\mathrm{X}$ del discurso y supuesto que $m_{B}$ pueda ser conocida. Aceptando que las palabras que no son borrosas en absoluto son las precisas, las minimales, $\mathrm{m}_{\mathrm{B}}$ dará el valor 0 cuando se aplique a las funciones con sólo los valores 0 y/o 1 que representan a los conjuntos clásicos. También se acepta que una palabra es maximal, o máximamente borrosa, cuando el valor que da para mp es 0.5 , ello proviene de suponer que se niega bajo la función de negación (fuerte) $1-\mathrm{x}$ cuyo punto fijo es 0.5 (Trillas, Eciolaza, 2015). Finalmente, se acepta que 'P es menos borrosa que Q' cuando,

- Entre 0 y 0.5 la función de pertenencia mP está por debajo de la mQ y, por tanto, más cerca de cero,

- Entre 0.5 y 1 la función de pertenencia mP está por encima de la mQ y, por tanto, más cerca de uno.

Obviamente, mP está más cerca de los valores 0 y 1 de lo que está mQ. Se tiene así una magnitud con las palabras cuya relación 'menos borrosa que' se obtiene de la forma anterior; se trata de un orden parcial entre las funciones de pertenencia y que tiene muchos minimales y un máximo.

Entonces, la función $m_{\mathrm{B}}$ es cualquiera que sea creciente respecto de tal orden y tome los valores 0 para las de los subconjuntos clásicos y 1 para la función constantemente igual a 0.5. Por lo tanto, el concepto de 'borrosidad' puede identificarse con las magnitudes escalares dadas por el conjunto de funciones $[0,1]^{\mathrm{X}}$ dotado de aquel orden parcial y una medida $\mathrm{m}_{\mathrm{B}}$; un concepto que puede trasladarse a las palabras imprecisas una vez han sido representadas por una medida de su significado cualitativo o función de pertenencia. El trabajo de De Luca y Termini, dio lugar al estudio de 'la borrosidad' (Fuzziness, en inglés) considerado como el objetivo de la lógica borrosa teórica y mostrando que puede verse como una parte científicamente domesticable del estudio de la vaguedad que, además, tiene multitud de aplicaciones prácticas.

\section{Agradecimientos}

Trabajo realizado con financiación del Fondo Europeo de Desarrollo Regional (FEDER) y el Ministerio de Economía y Competitividad español bajo el proyecto TIN2014-56622-C3-1-R. 


\section{Referencias}

ABELLANAS, P., (1963). Matemáticas para físicos e ingenieros. Romo.

ALSINA, C.; FRANK, MJ. SCHWEIZER, B. (2006). Associative Functions: Triangular Norms and Copulas. Worl Scientific.

ALSINA, C; TRILLAS, E; VALVERDE, Ll. (1983). “On Some Logical Connectives for Fuzzy Set Theory”. En Journal of Mathematical Analysis and Applications (93), 15-26.

ARREGUI, J., (1969). Concepto de magnitud escalar. En Cursillos sobre Didáctica Matemática, CSIC, II: 25- 37.

BICKERTON, D. (1990). Language and species. Chicago: University of Chicago Press.

BIRKHOFF, G. (1940). Lattice Theory. AMS Colloquium Publications.

CANTLON, J.F; PLATT, M; BRANNON, E.M (2009). Beyond the number domain. En Trends in Cognitive Science, vol. 13, n.2, 83-91.

DE LUCA, A.; TERMINI, S. (1972). "The concept of a Non-probabilistic entropy in the theory of fuzzy sets”. En Information and Control. (20), Issue 4, 301-312.

ELLIS, B., (1966). Basic Concepts of Measurement. Cambridge: Cambridge University Press.

HOWE, C: NUNES, T., BRYANT P. (2010). Intensive quantities: why they matter to developmental research. Br J Dev, Psychol. Jun (2), 307-29.

MENGER, K. (2007). Calculus: A Modern Approach. Dover (reedición de la $1^{\text {a }}$ Edición de 1955).

REY PASTOR, J., (1941). Curso cíclico de matemáticas. Lusy.

ROSS, J.R. (2004). Nouniness. En AARTS, B. (eds.) Fuzzy Grammar. A Reader. Oxford: University Press.

SAVAGE-RUMBAUGH, S.; LEWIN, R. (1994). Kanzi: the ape at the brink of the human mind. New York: John Wiley \& Sons.

SUGENO, M., (1974). Theory of Fuzzy Integrals and Its Applications. Tokio: Institute of Technology.

TRILLAS, E., (2017) On the Logos: A Naïve View on Ordinary Reasoning and Fuzzy Logic. Springer.

TRILLAS, E.; L. ECIOLAZA, (2015). Fuzzy Logic. Springer.

TRILLAS, E.; CASTRO, J.L (1995). An Essay on Vague Predicates as quantities, and associated Logics. En Proceedings EUSFIT’95. Vol. I: 18-22. 